\title{
Spatial Resonator Solitons
}

\author{
V. B. Taranenko, G. Slekys, C.O. Weiss \\ Physikalisch-Technische Bundesanstalt \\ 38116 Braunschweig, Germany
}

\begin{abstract}
Spatial solitons can exist in various kinds of nonlinear optical resonators with and without amplification. In the past years different types of these localized structures such as vortices, bright, dark solitons and phase solitons have been experimentally shown to exist. Many links appear to exist to fields different from optics, such as fluids, phase transitions or particle physics. These spatial resonator solitons are bistable and due to their mobility suggest schemes of information processing not possible with the fixed bistable elements forming the basic ingredient of traditional electronic processing. The recent demonstration of existence and manipulation of spatial solitons in semiconductor microresonators represents a step in the direction of such optical parallel processing applications. We review pattern formation and solitons in a general context, show some proof of principle soliton experiments on slow systems, and describe in more detail the experiments on semiconductor resonator solitons which are aimed at applications.
\end{abstract}

PACS 42.65.Tg, 47.54.+r, 42.65.Sf

\section{INTRODUCTION: A MULTIDISCIPLINARY VIEW AT PATTERN FORMATION AND SOLITONS}

In nonequilibrium nonlinear systems of all kinds patterns or "structures" are known to form. Their properties seem to be determined by a maximum dissipation principle. A system without structure is only able to dissipate as much energy as given by the microscopic dissipation processes. If then the system with structure (compatible with the system equations, parameters, boundary conditions and possibly initial conditions) has a higher dissipation, the structure will appear spontaneously e.g. through a modulational instability. This has been discussed at length in relation with the Bnard convection, but is easily found to be true in other fields.

In optics, when pumping a medium enough, then the microscopic relaxation processes do not provide the highest possible dissipation. As is well-known a coherent and structured and well ordered optical laser field can build which gives the system a much higher dissipation. If the pumping is increased, the laser emission may convert to a pulsed instead of a continuous emission in the form of regular or chaotic pulsing as described by the Lorenz Model. This pulsed laser emission is again increasing the system's capability to dissipate over the continuous emission. Such pulsing means a modulational instability in the temporal domain. Equally modulations or patterns appear in space, such as a hexagonal structure of the field in the emission cross section [1].

If noise sources act, the "coherent" structures permitting the high dissipation can appear stochastically. Examples are appearance of vortices in wave turbulence [2], vortex-antivortex generation in a noise driven Ginzburg Landau equation [3], and, evidently, phenomena such as earthquakes and stock market crashes [4]. The mentioned vortex-antivortex generation would seem to us to be a good picture for the spontaneous appearance of particleantiparticle pairs out of vacuum in quantum physics and we would think that it is also the proper model for the enigmatic phenomenon of $1 /$ f noise [5], particularly since this description contains the two best accepted explanations "self induced criticality" [6] and the often mentioned coupled oscillator model as special cases.

"Coherence", "structure" or "order" in space or time occurs thus in nonequilibrium systems to maximize the energy dissipation of the system. If one accepts such a principle (to which to our knowledge no counter examples are known) one can see this world as driven by a "pressure to dissipate" (where the pump evidently is the sun). In the way in which water pressed through a porous material or through rocks with cracks will form highly complex flow paths through the material, one can visualize by analogy the complex pattern formation in nature, culminating in biological structures, as forming under the pressure to dissipate and in the cracks and pores given by the boundary conditions in the world.

In this picture there is no need for a "plan" or "intention" to generate the complex structures which we find developed under the irradiation of the sun (absence of "teleological" elements). Everything is dictated by a simple extremum principle, which is "blind" in the sense that it can only find a nearest potential minimum. (And the system will directly head there even if "death" lurks in this minimum. There are good examples for such "suicidal" systems in laser physics [7].) A "teleological" element or "planning" and an "intention" appears only with the appearance of a brain, which can simulate the world and find out that there are several minima of which the nearest may not be the deepest (or that at the nearest minimum "death" may lurk). Even then it appears that the teleological element seems to be the exception. Even among the most complex structure, the human species, most decisions are done unconsciously. 
It is evidently one of the goals of nonlinear physics to clarify and understand the origin of life and of biological structures. Although the path from simple periodic patterns, appearing as a modulational instability, and spatial solitons, to structures as complex as biological ones would seem exceedingly long at first glance, this may not be so at second glance.

A simple periodic pattern such as a hexagonal field structure in the cross section of a nonlinear resonator can disintegrate into spatial resonator solitons [8]. A closer examination of these structures shows many common traits with biological structures.

Spatial solitons in nonlinear dissipative resonators

1) are mobile (allowing them to move to the point in space where they are most stable i.e. find the best "living conditions" (i.e. they can search around for food));

2) they possess a "metabolism": they have internal energy flows and spatially varying dissipation, which stabilize them;

3) they can "die": when the field sustaining them ("food") is reduced, they will extinguish (die). And even a re-increase of the field to a strength which allowed the solitons to exist before will not reignite them, because of their bistability (death is irreversible);

4) they can multiply ("self-replicate") by repeated splitting [9].

There are further common traits of dissipative resonator solitons and biological structures, among them the fact that both exist only at medium nonlinearities and not at large or small nonlinearities (see below).

Consequently one could very well conjecture that the simplest biological ("live") structures are not viruses (as commonly taught) but dissipative solitons. This, by the way, shedding a completely new light on the question of what biological life is: life is usually ascribed to chemistry-based structures (with carbon the central compound). Whereas if one takes the analogies above seriously, "life" is not bound to chemistry but constitutes itself as organizational "forms" of matter or fields. Thus the probability of finding "live" structures is much larger than finding "life" only in its form bound to carbon or chemistry.

These analogies of dissipative solitons with biological structures are one of the reasons for which one might want to study spatial solitons. Furthermore these solitons are all by definition bistable i.e. they constitute natural carriers of information. As opposed to the information carrying elements in electronic information processing, they are mobile and would therefore perhaps permit processing functions beyond the reach of electronic computing. We have recently studied the "aggregation" of spatial solitons and find again common traits with biology. Considering that these "aggregations" of information carriers constitute something like a "brain" (a collection of information-carrying elements, bound loosely to one another, capable of exchanging information among the elements) there is another motivation for the study: One might hope, in the way there are analogies between solitons and simple biological ("live") structures, to find operation principles of the brain by studying the structure and properties of the "aggregations" of spatial solitons.

Optics and particularly resonator optics suggest themselves for the investigations, because of possible photonic applications such as parallel processing, urgently awaited already e.g. in telecom. For this reason we have conducted in the past a series of "proof of existence"experiments on resonator solitons as well as of their manipulation and properties [10]. Presently spatial resonator solitons in semiconductor micro-resonators are investigated, which are most likely best suited for applications.

\section{PROOF OF EXISTENCE EXPERIMENTS OF RESONATOR SOLITONS WITH SLOW MATERIALS}

Spatial resonator solitons are self-formed localized structures, which are free to move around. We have investigated several types:

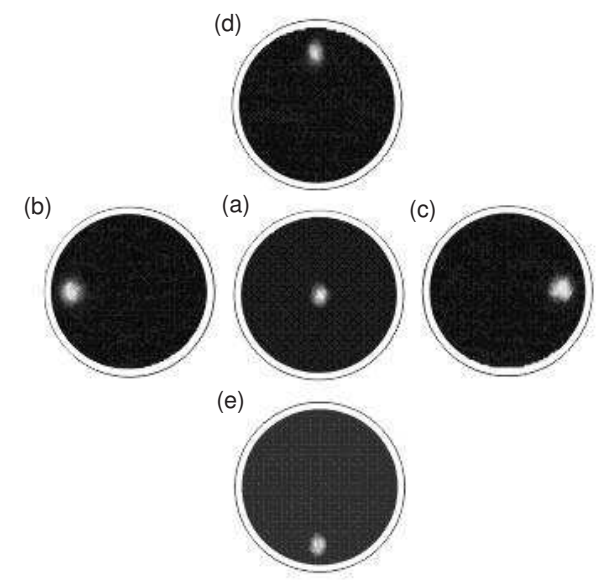

FIG. 1. In a laser with internal nonlinear absorber (bacterio-rhodopsin) a soliton can be written in arbitrary places in the resonator cross section. The resonator used is degenerate for all transverse modes to allow any arbitrary field configuration to be resonant.

Vortices occur in resonators with a gain medium without phase preference, such as laser resonators. They are helical defects in the wavefront and possess therefore, strictly speaking, "tristability": right- as well as left-handed vortices coexist with the wave without defect. We have given an overview of laser vortices in [10] including examples of their dynamics in Internet films 
[11]. Vortices were until now not studied with regard to their usefulness as binary information carriers (except for some basic experiments relating to pattern recognition with lasers [12]).

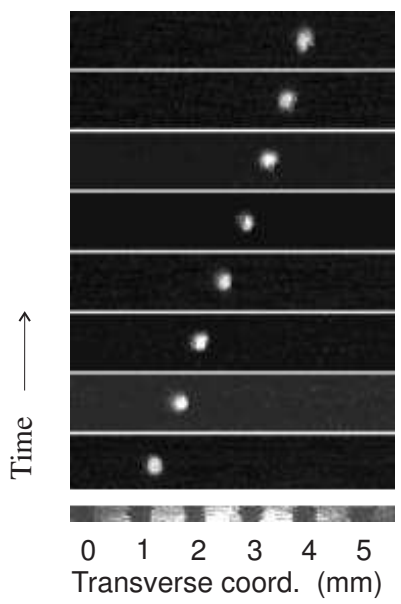

FIG. 2. A soliton can be made to move across the resonator cross section by a phase gradient in the resonator.

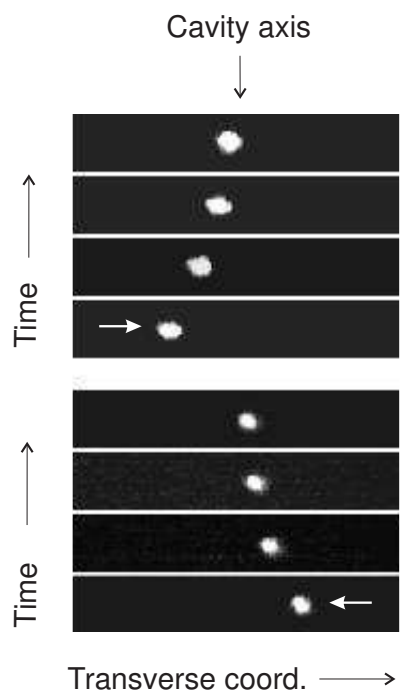

FIG. 3. In a resonator with a "phase trough" at the center a soliton is drawn from all sides to the resonator axis and is trapped there.

Bright solitons are more reminiscent of the original idea of optical information processing namely the use of optically bistable resonators. They have therefore been considered more closely for such tasks. In order to convince oneself that such bright solitons in bistable resonators exist generally, we conducted experiments with nonlinear media with slow response - for the purpose of being able to observe the phenomena (which entail $2 \mathrm{D}$ space-time dynamics) on a convenient time scale. As op- posed to semiconductors which have characteristic times of ps to ns, e.g. photorefractive media and nonlinear absorbers like bacterio-rhodopsine [13] have time constants of $10 \mathrm{~ms}$ to $1 \mathrm{~s}$, quite suitable for recording with ordinary video equipment. In general a resonator containing only such a non-linear absorber is too lossy for bistability. Therefore we used resonators containing additionally gain elements to compensate for the losses. A system like a laser with nonlinear absorber results which is well suited to study these transverse effects such as spatial modulational instabilities, patterns and solitons.

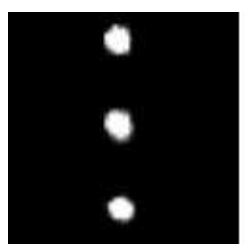

(a)

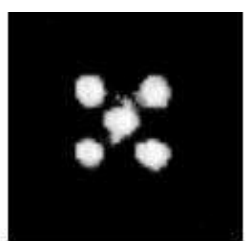

(b)

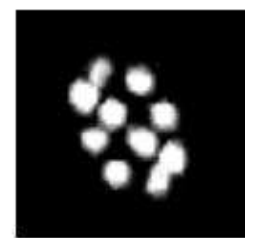

(c)
FIG. 4. Larger number of stationary bright solitons coexisting.

The first of such experiments yielded evidence of the existence of spatial solitons [14]. In refined experiments we showed the existence, the bistability and the manipulations of bright soliton in this system [15]. FIG. 1 shows that the solitons can be "ignited" anywhere in the cross section of the resonator. FIG. 2 shows the motion of a bright soliton in a phase gradient, and FIG. 3 shows the "trapping" of a bright soliton in a "phase-trough". These are functions as they are required for applications. FIG. 4 shows that large numbers of such bright solitons can exist at the same time [9], equally as necessary for technical applications.

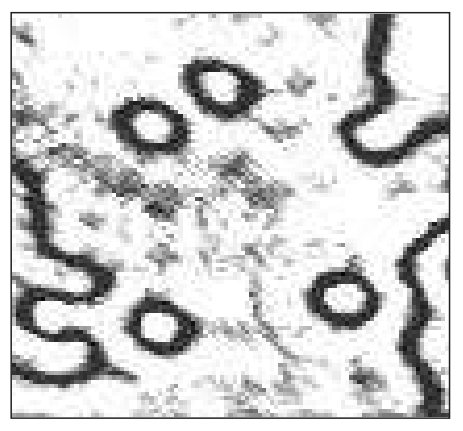

FIG. 5. Phase solitons coexisting with non stationary phase fronts. At the fronts and surrounding the soliton the phase of the field changes by 180 degrees producing by interference the dark ring surrounding the solitons and the dark fronts at the phase-domain boundaries.

As an interesting feature of these solitons it was found [16] that these solitons have quantized velocity magni- 
tudes (including velocity magnitude zero). Although the velocity magnitudes of such solitons are fixed, their direction of motion is completely free, and varies e.g. under the action of noise.

It may be mentioned that in resonators with phasesensitive gain, such as gain supplied by 4 -wave-mixing or degenerate parametric mixing, solitons which concern only the phase structure of the field (phase-solitons) were predicted [17] and experimentally observed [18]. See FIG. 5.

\section{SEMICONDUCTOR RESONATOR SOLITONS}

To make bright solitons suitable for technical applications, fast materials have to be used. We found that the optimum system concerning speed of response and nonlinearity is the semiconductor micro-resonator. This is a resonator of $\sim 1$ wavelength length which provides the shortest conceivable resonator response time of $100 \mathrm{fs}$ 1 ps. The finesse of these resonators has to be around 100 - 200 to provide bistability together with a nonlinear medium. For the latter a semiconductor slice of the length of the resonator $(\sim 1 \lambda)$ is suitable, and we stress that the response time of semiconductor material is well matched to that of the resonator. Importantly: it makes no sense to use "faster" materials than semiconductors in such resonators. Since the small length of the resonator provides a response time which is at the conceivable limit for optical resonators, a faster nonlinear material does not decrease the system response time. However, since higher speed comes at the cost of a smaller nonlinearity, a faster material would require unnecessarily high light intensities. Thus the $\sim 1 \lambda$ microresonator combined with a semiconductor material as the nonlinear ingredient represents the optimum in terms of response time and required light intensity. This structure is incidentally the structure of VCSELs (vertical cavity surface emitting laser) which during the last years have already been developed to a certain degree of perfection. For the existence of spatial solitons in such resonators absorbing ("passive") as well as population-inverted ("active") material is suitable. Thus a direct connection with VCSEL technology exists.

FIG. 6 shows the structure of the nonlinear resonator. Its length is $\sim 1 \lambda$ while the transverse size is typically $5 \mathrm{~cm}$. The short length and wide area of this microresonator permit only one longitudinal mode (FIG. 7 (a)) while allowing an enormous number of transverse modes so that a very large number of spatial solitons can coexist. The resonator is obviously of the plane mirror type, implying frequency degeneracy of all transverse modes and thus allowing arbitrary field patterns to be resonant inside the resonator. This is another prerequirement for existence and manipulability of spatial solitons.

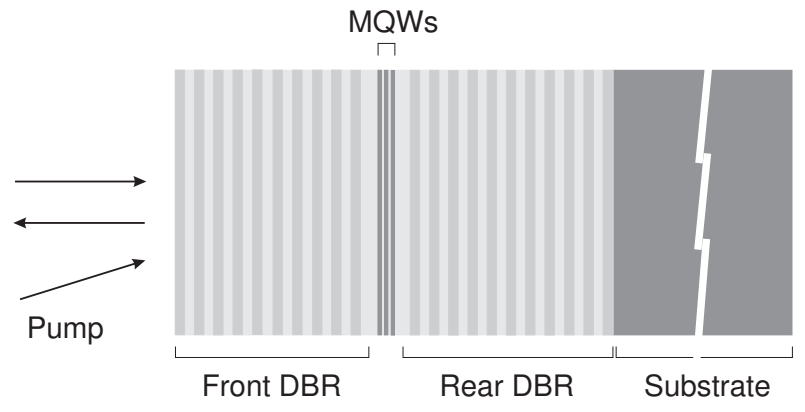

FIG. 6. Schematic of semiconductor microresonator consisting of two plane distributed Bragg reflectors (DBR) and multiple quantum wells (MQW). Observation is in reflection.
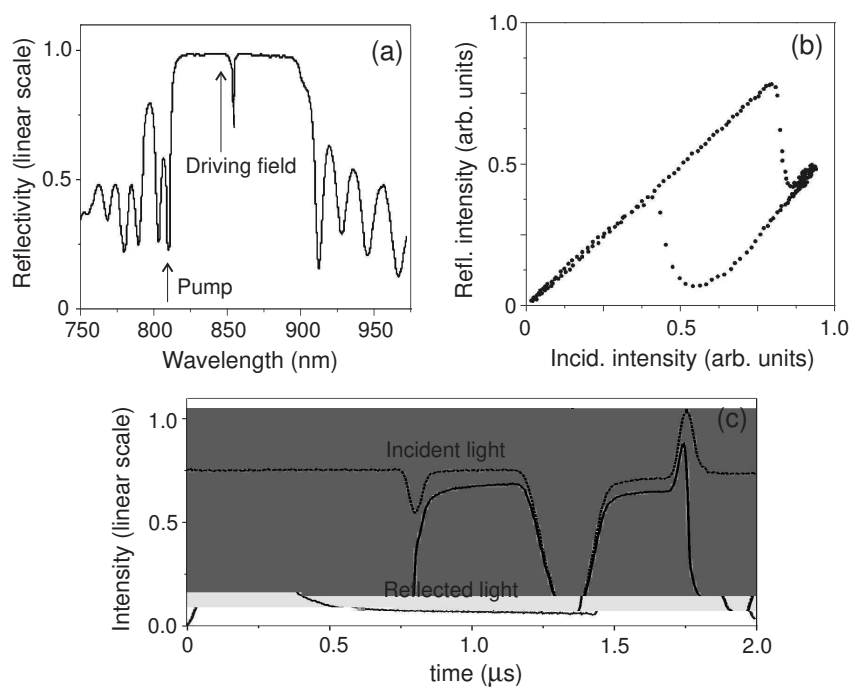

FIG. 7. Semiconductor microresonator reflectance spectrum (a), typical bistability loop in reflection (b) and dynamics of switching (c). Arrows mark the driving field that is detuned from the resonator resonance and the pump field that is tuned to be coupled into the resonator through one of the short-wavelength interference notches of the resonator reflectance spectrum. (c) shows that by short positive (negative) pulses the resonator can be switched from one branch of bistability loop to the other.

The resonator soliton existence is closely linked with the plane wave resonator bistability (FIG. 7 (b)) caused by longitudinal nonlinear effects: the nonlinear changes of the resonator length (due to nonlinear refraction changes) and finesse (due to nonlinear absorption changes) [19]. The longitudinal nonlinear effects combined with transverse nonlinear effects (such as selffocusing) can balance diffraction and form resonator solitons. Generally these nonlinear effects can cooperate or act oppositely, with the consequence of reduced soliton stability in the latter case. 


\section{A. Model and numerical analysis}

As a guide for the experiments we use a phenomenological model of a driven wide area multiquantum well (MQW)-semiconductor microresonator similar to [20,21]. The optical field $A$ inside the resonator is described in the mean-field approximation [22]. The driving incident field $A_{\text {in }}$ is assumed to be a stationary plane wave. Nonlinear absorption and refractive index changes induced by the intracavity field in the vicinity of the MQW-structure band edge are assumed to be proportional to the carrier density $N$ (normalized to the saturation carrier density). The equation of motion for $N$ includes optical pumping $P$, carrier recombination and diffusion. The resulting coupled equations describing the spatio-temporal dynamics of $A$ and $N$ have the form:

$$
\begin{array}{r}
\partial A / \partial t=A_{\text {in }}-\sqrt{T} A\{[1+C \operatorname{Im}(\alpha)(1-N)]+ \\
\left.+i\left(\theta-C \operatorname{Re}(\alpha) N-\nabla_{\perp}^{2}\right)\right\}, \\
\partial N / \partial t=P-\gamma\left[N-|A|^{2}(1-N)-d \nabla_{\perp}^{2} N\right],
\end{array}
$$

where $C$ is the saturable absorption scaled to the resonator transmission $T$ ( $T$ is assumed to be small since the mirror reflectivity is typically $\geq 0.995) . \operatorname{Im}(\alpha)(1-N)$ and $\operatorname{Re}(\alpha) N$ describe the absorptive and refractive nonlinearities, respectively. $\theta$ is the detuning of the driving field from the resonator resonance. $\gamma$ is the photon lifetime in the resonator normalized to the carrier recombination time. $d$ is the diffusion coefficient scaled to the diffraction coefficient, and $\nabla_{\perp}^{2}$ is the transverse Laplacian.

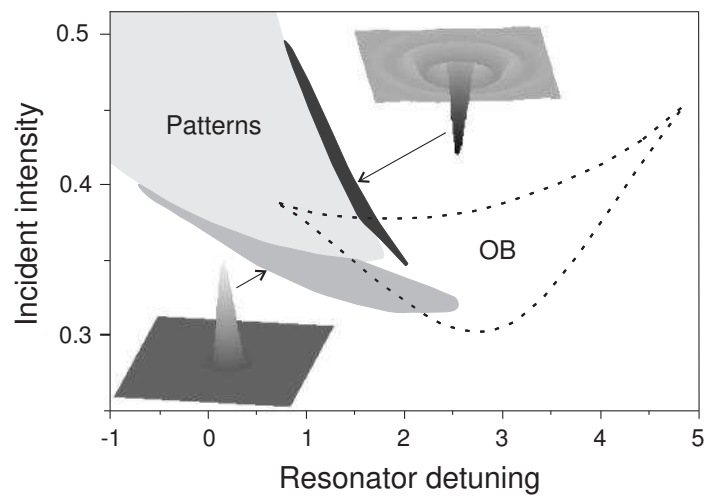

FIG. 8. Numerical solutions of Eq.(1) for unpumped and mixed absorptive/self-focusing case. Area limited by dashed lines is optical bistability domain for plane waves. Shaded areas are domains of stability for bright/dark solitons and patterns. Insets are bright and dark solitons in 3D representation.

Linear effects in the resonator are spreading of light by diffraction, and carrier diffusion (terms with $\nabla_{\perp}^{2}$ in
(1)). The material nonlinearity that can balance this linear spreading can do this in various ways. It has a real (refractive) and imaginary (dissipative) part and can act longitudinally and transversely. The nonlinear changes of the resonator finesse (due to nonlinear absorption change) and length (due to nonlinear refractive index change) constitute longitudinal nonlinear effects, also known under the name nonlinear resonance [17]. The transverse effects of the nonlinear refractive index can be self-focusing (favorable for bright and unfavorable for dark solitons) and self-defocusing (favorable for dark and unfavorable for bright solitons). Absorption (or gain) saturation (bleaching) leading to nonlinear gain guiding (in laser parlance), is a transverse effect. Longitudinal and transverse effects can work oppositely, or cooperate.

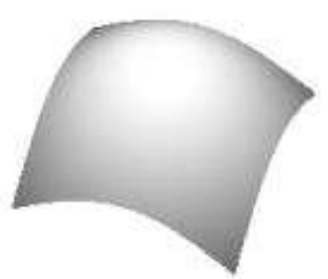

(a)

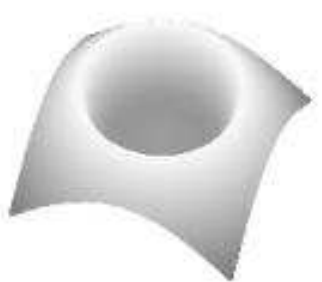

(b)
FIG. 9. Maxwellian switched domain in reflection (b) numerically calculated for parameters corresponding to the plane-wave OB area in FIG. 8 and for Gaussian profile (a) of the driving beam.

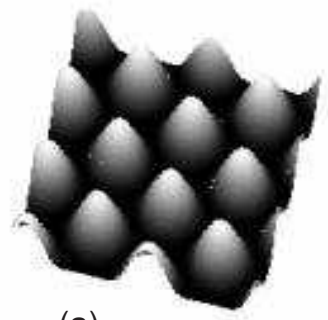

(a)

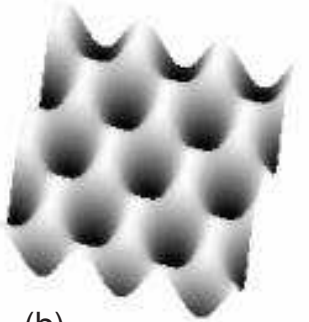

(b)
FIG. 10. Bright- (a) and dark- (b) spot-hexagons numerically calculated for parameters corresponding to the Pattern existence domain of FIG. 8. Driving light intensity increases from (a) to (b).

There are two principal external control parameters: the driving field intensity $\left|A_{\text {in }}\right|^{2}$ and the resonator detuning $\theta$. FIG. 8 shows typical existence domains (in coordinates $\theta,\left|A_{\text {in }}\right|^{2}$ ) for all possible structures (patterns, bright and dark spatial solitons) and plane-wave bistability as calculated from (1) for the case of a mixed absorptive/dispersive nonlinearity. At large resonator detuning the intracavity field is transversely homogeneous and stable. If in this case the driving beam has a Gaussian profile 
(FIG. 9 (a)) and its amplitude in the maximum exceeds the switching-on threshold then the part of the beam cross section which is limited by so called Maxwellian intensity [23] is switched, thus forming a dark switched domain in reflection (FIG. 9 (b)).

For small resonator detuning and for driving intensities not quite sufficient for reaching the resonance condition for the whole resonator area, the system "chooses" to distribute the light intensity in the resonator in isolated spots where the intensity is then high/low enough to reach the resonance condition, thus forming bright/dark patterns (FIG. 10). Instead of saying "the system chooses" one would more mathematically express this by describing it as a modulational instability. The detuned plane wave field without spatial structure with intensity insufficient to reach the resonance condition is unstable against structured solutions. According to our numerical solutions of (1) a large number of such structured solutions coexist and are stable (see e.g. patterns in FIG. 18).

The bright/dark soliton structures (inset in FIG. 8) can be interpreted as small circular switching fronts, connecting two stable states: the low transmission and the high transmission state. Such a front can in 2D surround a domain of one state. When this domain is comparable in diameter to the "thickness" of the front, then each piece of the front interacts with the piece on the opposite side of the circular small domain, which can lead, particularly if the system is not far from a modulational instability (see FIG. 8, Patterns), to a stabilisation of the diameter of the small domain. In this case the small domain is an isolated self-trapped structure or a dissipative resonator soliton.

\section{B. Experimental arrangement}

FIG. 11 shows the optical arrangement for the semiconductor soliton experiments, and in particular for their switching on or off. The semiconductor microresonator consists of a MQW (GaAs/AlGaAs or GaInAs/GaPAs) structure sandwiched between high-reflectivity $(\geq 0.995)$ DBR-mirrors (FIG. 6) used at room temperature. The microresonator structures were grown on GaAs substrates by a molecular beam epitaxy technique that allows growing MQW structures with small radial layer thickness variation. The best sample used in our experiments has only $\sim 0.3 \mathrm{~nm} / \mathrm{mm}$ variation of the resonator resonance wavelength over the sample cross section of 5 cm diameter.

The driving light beam was generated by either a tunable (in the range 750-950 nm) Ti:Sa laser or a singlemode laser diode $(\sim 854 \mathrm{~nm})$, both emitting continuously. For experimental convenience and to limit thermal effects, the experiments are performed within a few microseconds, by admitting the light through an acustooptical modulator. A laser beam of suitable wavelength is focused onto the microresonator surface in a spot of $\sim 50 \mu \mathrm{m}$ diameter, thus providing quite large Fresnel number $(\geq 100)$.

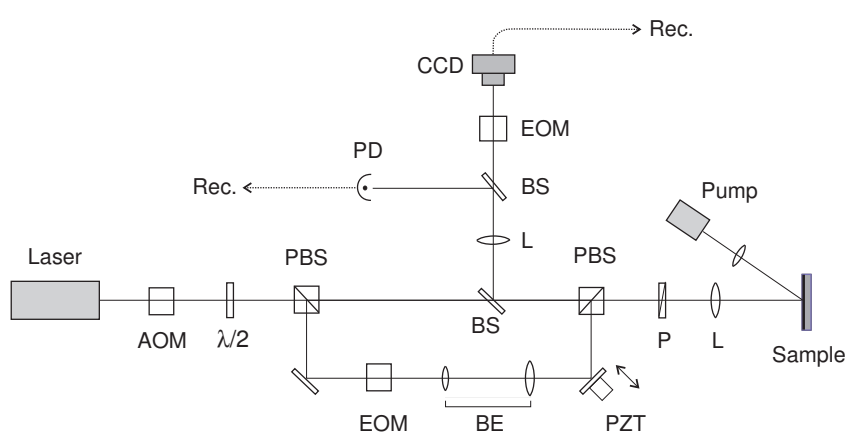

FIG. 11. Experimental setup. Laser: Ti:Sa (or diode) laser, AOM: acousto-optic modulator, $\lambda / 2$ : halfwave plate, PBS: polarization beam spliters, EOM: electro-optical amplitude modulators, BE: beam expander, PZT: piezo-electric transducer, P: polarizer, L: lenses, BS: beam splitters, PD: photodiode. In some cases an optical pump is used ("Pump"), see text.

Part of the laser light is split away from the driving beam and is superimposed with the main beam in a Mach-Zehnder interferometer arrangement, to serve as a writing/erasing (address) beam. This beam is tightly focused and directed to some particular location in the illuminated area to create or destroy a spatial soliton. The switching light is opened for a few nanoseconds using an electro-optic modulator. For the case of incoherent switching the polarization of the address beam is perpendicular to that of the main beam to avoid interference. For the case of coherent switching the polarizations are parallel and a phase control of the switching field is always needed: for switching on as well as switching off a soliton. One of the interferometer mirrors can be moved by a piezo-electric element to control the phase difference between the background light and the address light.

Optical pumping of the MQW-structures was done by a multi-mode laser diode or a single-mode Ti:Sa laser. To couple the pump light into the microresonator the pump laser wavelength was tuned into the short wavelength reflection minimum as shown in FIG. 7 (a).

The observations are done in reflection (because the GaAs substrate is opaque) by a CCD camera combined with a fast shutter (another electro-optic modulator), which permits to take nanosecond snapshots at a given time, of the illuminated area on the resonator sample. Recording movies on the nanosecond time scale is also possible. To follow the intensity in time in certain points (e.g. at the location of a soliton) a fast photodiode can be imaged onto arbitrary locations within the illuminated area. 


\section{Results and discussions}

Switched (dark) domains exist for small negative resonator detuning in the vicinity of the absorption band edge. The switching front surrounding these domains is defined by the spatial profile of the driving beam. The switching front is located where the driving intensity equals the Maxwellian intensity. FIG. 12 shows snapshots of various switched domains demonstrating that they have the same shapes as the driving beams. When changing the intensity of the driving beam in time the fronts around the switched domains follow obviously an equiintensity contour of the incident light (FIG. 13).

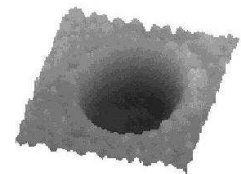

(a)

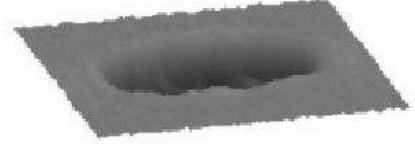

(b)
FIG. 12. Dark switched domains observed at small (negative) resonator detuning for round (a) and oval (b) driving beam shapes.

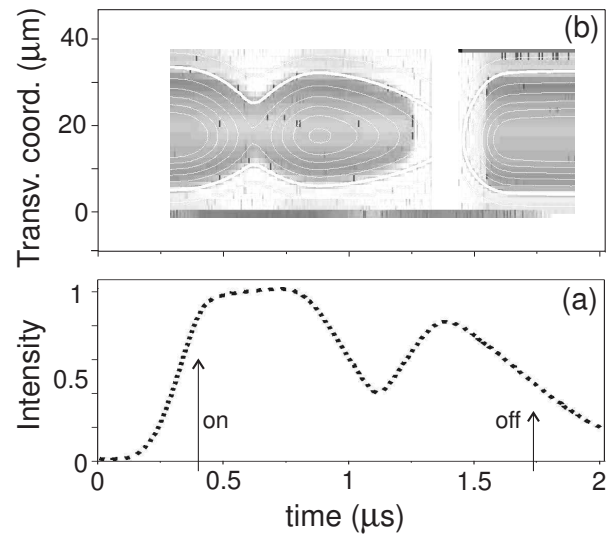

FIG. 13. Incident driving light intensity, center of Gaussian beam (a) and reflectivity of sample (on diameter of circular driving beam cross section) along with equiintensity contours of incident light (b) demonstrating that borders of dark switched area follow one of the equiintensity contours (heavy line).

In contrast to these dark Maxwellian switched domains, spatial patterns and solitons are self-sustained objects independent of boundary conditions or a beam profile and can be both bright and dark. FIG. 14 shows snapshots of bright and dark small $(\sim 10 \mu \mathrm{m})$ round spots (solitons), at large (negative) resonator detuning, whose shape/size is independent of the shape/intensity of the driving beam [24]. This independence of boundary con-

ditions allows to distinguish between patterns and solitons on the one side and Maxwellian switched domains on the other side.

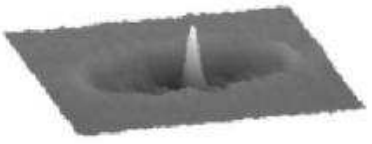

(a)

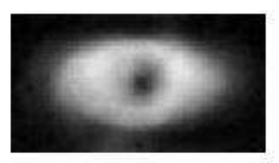

(c)

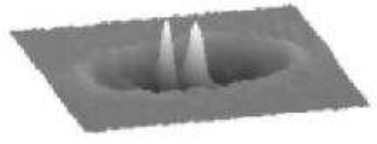

(b)

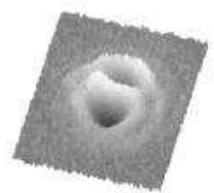

(d)

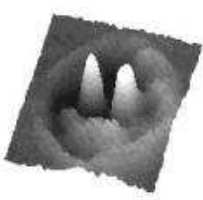

(e)
FIG. 14. Bright- and dark-spot switched structures observed experimentally at large (negative) resonator detuning. The independence of the structures of the driving beam shape shows that the structures are self-localized (patterns or spatial solitons).

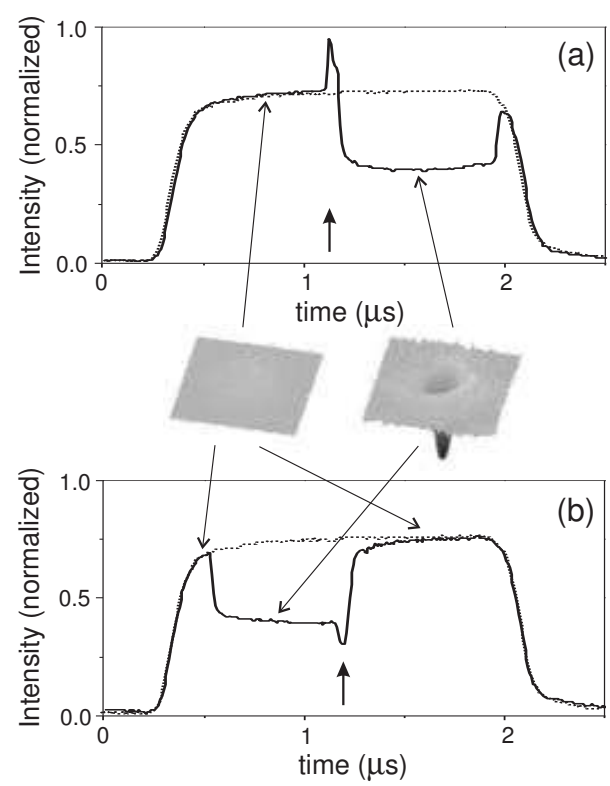

FIG. 15. Recording of coherent switching-on (a) and switching-off (b) of a bright soliton. Heavy arrows mark the application of switching pulses. Dotted traces: incident intensity; solid traces: reflected intensity at center of soliton. The insets show intensity snapshots, namely unswitched state (left) and soliton (right). Details see text.

Resonator solitons can be written and erased by focused optical (coherent) pulses independent of other bright (dark) spots (solitons).

FIG. 15 shows how solitons can be switched by light coherent with the background light [25]. FIG. 15 (a) shows switching a bright soliton on. The driving light inten- 
sity is chosen slightly below the spontaneous switching threshold. At $t \approx 1.2 \mu$ s the writing pulse is applied. It is in phase with the driving light, as visible from the constructive interference. A bright soliton results, showing up in the reflected intensity time trace as a strong reduction of the intensity. FIG. 15 (b) shows switching a soliton off. The driving light is increased to a level where a soliton is formed spontaneously. The address pulse is then applied in counterphase to the driving light, as visible from the destructive interference. The soliton then disappears, showing up in the reflected intensity time trace as reversion to the incident intensity value. The FIG. 15 insets show 2D snapshots before and after the switching pulses for clarity.

Thus depending on conditions we find switched domains, patterns and bright and dark resonator solitons. Periodic patterns can be distinguished from collections of solitons by the mutual independence of the latter.

To find the most stable resonator solitons for applications one can play with the nonlinear (absorptive/dispersive) material response by choice of the driving field wavelength and intensity, with the resonator detuning, and finally with the carrier population inversion (when using pumping). We recall that all nonlinearities change their sign at transparency i.e. at the point where the valence- and the conduction band populations are equal. Going from below transparency (absorption) to above transparency (population inversion, producing light amplification), nonlinear absorption changes to nonlinear gain, self-focusing changes to self-defocusing and vice versa, and decrease of optical resonator length with intensity changes to increase (and vice versa). The population of the bands can be controlled by pumping $(P$ in (1)) i.e. transferring electrons from the valence band to the conduction band. We do this by optical excitation [26], with radiation of a wavelength shorter than the band edge wavelength. If the structures were suited to support electrical currents (i.e. if it were a real VCSEL-structure) pumping could evidently be effected by electrical excitation.

\section{Illumination below bandgap (defocusing nonlinearity)}

Working well below the bandgap (in the dispersive/defocusing limit) with the driving field wavelength $\sim 30 \mathrm{~nm}$ longer than the band edge wavelength we observe spontaneous formation of hexagonal patterns (FIG. 16). The hexagon period scales linearly with $\theta^{-1 / 2}[27]$ indicating that the hexagons are formed by the tilted-wave mechanism [28], which is the basic mechanism for resonator pattern formation [29]. Dark-spot hexagons (FIG. 16 (a)) convert to bright-spot hexagons (FIG. 16 (b)) when the driving intensity increases. This is in qualitative agreement with numerical simulations (FIG. 10).

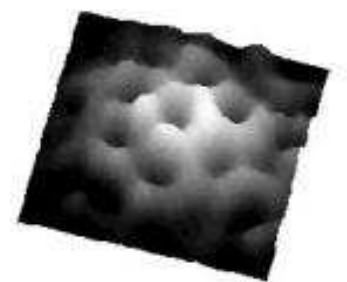

(a)

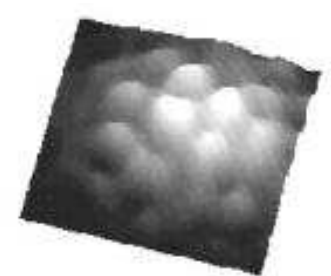

(b)
FIG. 16. (a) Bright (dark in reflection) and (b) dark (bright in reflection) hexagonal patterns for the dispersive/defocusing case. Driving light intensity increases from (a) to (b).

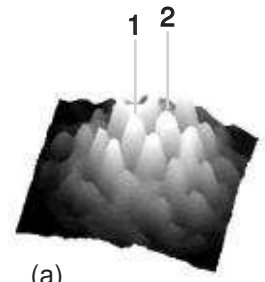

(a)

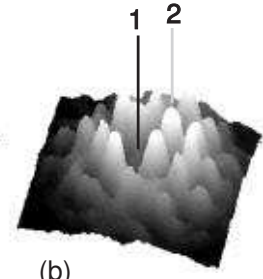

(b)

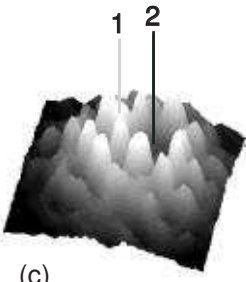

(c)
FIG. 17. Switching-off of individual spots of a hexagonal structure with address pulses aimed at different bright spots (marked 1 and 2) of the pattern. (a)

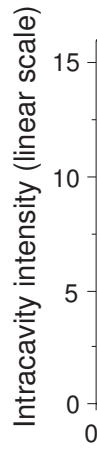

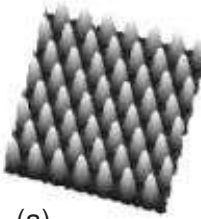

(c)

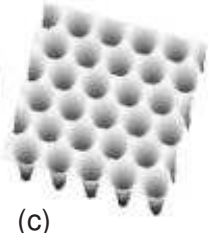

(b)

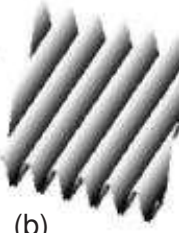

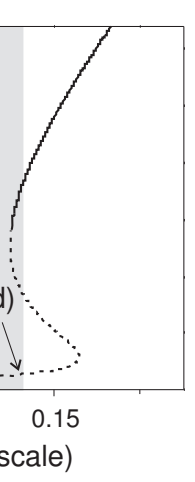

FIG. 18. Numerical solutions of Eq.(1) for intracavity light intensity as function of incident intensity: homogeneous solution (dashed line marks modulationally unstable part of the curve) and patterns (a-d). Shaded area marks existence range for dark-spot hexagons.

At high driving intensity we find that the bright spots in such hexagonal patterns can be switched independently from one another by focused optical (incoherent) pulses [27]. FIG. 17 shows the experimental results. 
FIG. 17 (a) shows the hexagonal pattern formed. The focused light pulse can be aimed at individual bright spots such as the ones marked "1" or "2". FIG. 17 (b) shows that after the switching pulse was aimed at "1", spot "1" was switched off. FIG. 17 (c) shows the same for spot "2". We note that in these experiments we speak of true logic switching: the spots remain switched off after the switching pulse, (if the energy of the pulse is sufficient, otherwise the bright spot reappears after the switching pulse). These observations of local switching indicate that these hexagonal patterns are not coherent patterns. The individual spots are spatial solitons: they are independent, even at this dense packing where the spot distance is about the spot size.

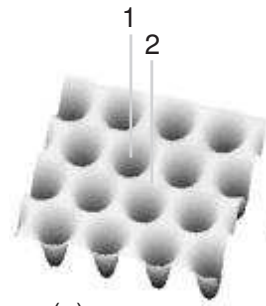

(a)

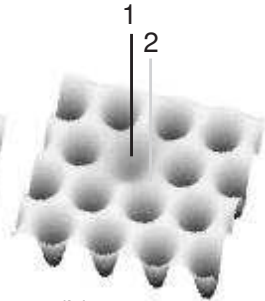

(b)

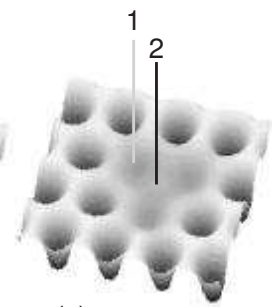

(c)
FIG. 19. Stable hexagonal arrangements of dark spatial solitons: (a) without defects, (b) with single-soliton defect, (c) with triple-soliton defect. Parameters as for FIG. 18 (d). Compare with the experiment FIG. 17 and [27].

These experimental findings can be understood in the frame of the model (1) [8]. FIG. 18 shows the bistable plane wave characteristic of the semiconductor resonator for conditions roughly corresponding to the experimental conditions. At the intensities marked (a) to (d) patterned solutions exist.

The pattern period in FIG. 18 (a) scales again linearly with $\theta^{-1 / 2}$ due to the tilted wave mechanism: When the driving field is detuned, the resonance condition of the resonator cannot be fulfilled by plane waves travelling exactly perpendicularly to the mirror plane. However, the resonance condition can be fulfilled if the wave plane is somewhat inclined with respect to the mirror plane (the tilted wave mechanism [28]). The system chooses therefore to support resonant, tilted waves. FIG. 18 (a) is precisely the superposition of six tilted waves that support each other by (nonlinear) 4-wave-mixing. The pattern period corresponds to the resonator detuning as in the experiment for structures FIG. 16 (a). In this pattern (FIG. 18 (a)) the bright spots are not independent. Individual spots cannot be switched as in the experiment FIG. 17.

On the high intensity pattern FIG. 18 (d) the pattern period is remarkably different from FIG. 18 (a) even though the (external) detuning is the same. This is indication that the internal detuning is smaller and means that the resonator length is nonlinearly changed by the intensity-dependent refractive index (nonlinear resonance). From the ratio of the pattern periods of FIG. 18 (a) and (d) one sees that the nonlinear change of detuning is about half of the external detuning. That means the nonlinear detuning is by no means a small effect. This in turn indicates that by spatial variation of the resonator field intensity the detuning can vary substantially in the resonator cross section. In other words, the resonator has at the higher intensity a rather wide freedom to (self-consistently) arrange its field structure. One can expect that this would allow a large number of possible stable patterns between which the system can choose - or which are chosen by initial conditions.

FIG. 19 shows that at the high intensity corresponding to FIG. 18 (d) the model (1) allows to reproduce the experimental findings on switching individual bright spots. FIG. 19 (a) is the regular hexagonal pattern, at high intensity. FIG. 19 (b) shows the field with one bright spot switched off as a stable solution and FIG. 19 (c) shows a triple of bright spots switched off as a stable solution, just as observed in the experiments [8].

Thus while FIG. 18 (a) is a completely coherent space filling pattern, FIG. 18 (d) is really a cluster of (densest packed) individual dark solitons. The increase of intensity from (a) to (d) allows the transition from extended "coherent" patterns to localized structures, by the increased nonlinearity, which gives the system an additional internal degree of freedom. We note that the transition from the coherent low intensity pattern to the incoherent higher intensity structure proceeds through stripe patterns as shown in FIG. 18 (b) [8]. For the intensity of FIG. 18 (c) the individual spots are still not independent, corresponding to the experiment FIG. 16 (b).

\section{Illumination near bandgap (absorptive/defocusing nonlinearity)}

Working at wavelengths close to the band edge we find bright and dark solitons (FIG. 20), as well as collections of several spots (FIG. 14 (b),(d),(e)) (all independent on the illumination beam profile).

The nonlinearity of the MQW structure near the band edge is predominantly absorptive. For comparing with calculations we can therefore in the first approximation neglect the refractive part of the complex nonlinearity in the model equations (1) and describe the nonlinear medium as a saturable absorber. Numerical simulations for this case (FIG. 21) confirm existence of both bright and dark resonator solitons as they are observed in the experiment (Figs 14, 20). We can contrast these purely dissipative resonator spatial solitons with propagating spatial solitons (in a bulk nonlinear material) [30]: the latter can not be supported by saturable absorption. 


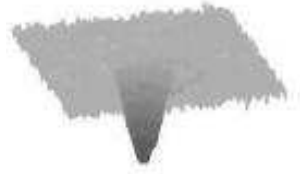

(a)

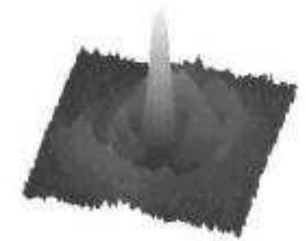

(b)
FIG. 20. Dark and bright solitons experimentally observed near bandgap.

FIG. 22 shows details of the spontaneous formation of such bright solitons as in FIG. 14 (c) and FIG. 20 (a). As discussed in [24] material heating leads in this case to a slow spontaneous formation of solitons, associated with the shift of the semiconductor band edge by temperature [31]. In FIG. 22 at $t \approx 1.3 \mu$ s (arrow) the resonator switches to high transmission (small reflection). The switched area then contracts slowly to the stable structure FIG. 22 (b), which is existing after $t \approx 3.0 \mu \mathrm{s}$.

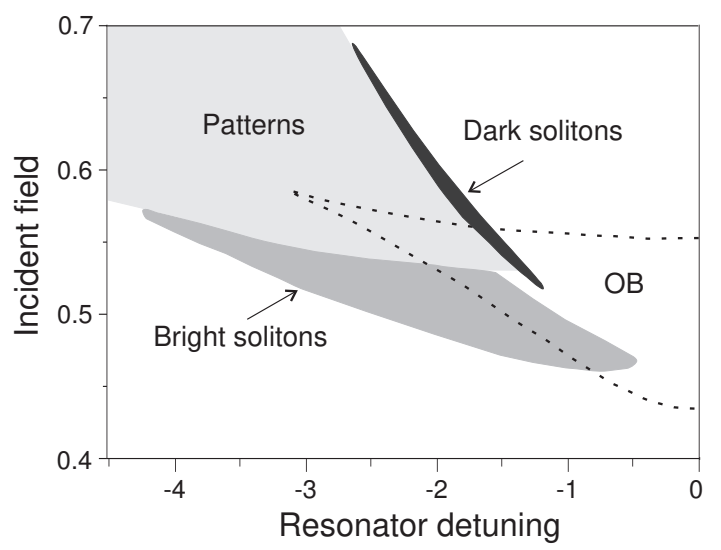

FIG. 21. Numerical solutions of Eq.(1) for unpumped $(P=0)$, absorptive $(\operatorname{Re}(\alpha)=0)$ case. Area limited by dashed lines is optical bistability domain for plane waves. Shaded areas are domains of existence of bright and dark solitons.

After the resonator has switched to low reflection its internal field and with it the dissipation is high. A rising temperature $\Delta T$ decreases the band gap energy [31] $\left(E_{\mathrm{g}} \approx E_{\mathrm{go}}-\alpha \Delta T\right)$ and therefore shifts the bistable resonator characteristic towards higher intensity. Thus the basin of attraction for solitons which is located near the plane wave switch-off intensity (see locations of the existence domains for the bright solitons and the plane wave bistability in FIG. 21) is shifted to the incident intensity, whereupon a soliton can form. Evidently for different parameters the shift can be substantially larger or smaller than the width of bistability loop, in which case no stable soliton can appear. In consequence, we note that in absence of thermal effects (good heat-sinking of sample) solitons would not appear spontaneously, but would have to be switched on by local pulsed light injection.
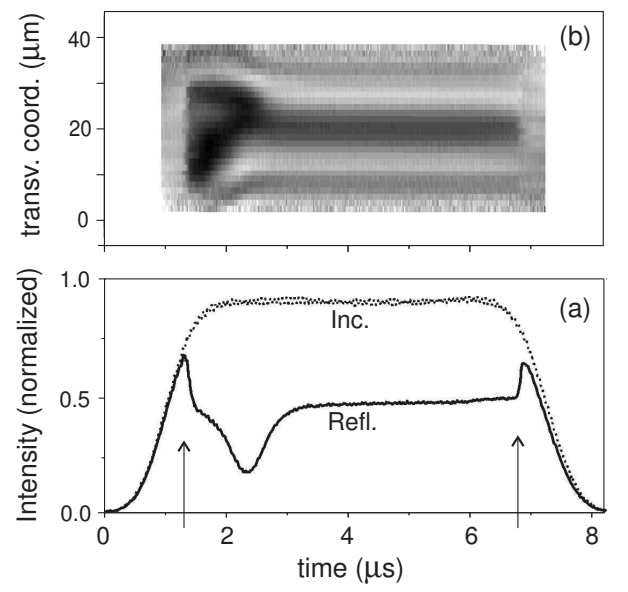

FIG. 22. Bright soliton formation below bandgap. Reflectivity on a diameter of the illuminated area as a function of time (b). Intensity of incident (dotted) and reflected (solid) light, at the center of the soliton as a function of time (a). Arrows mark the switch-on and -off. Details see text.
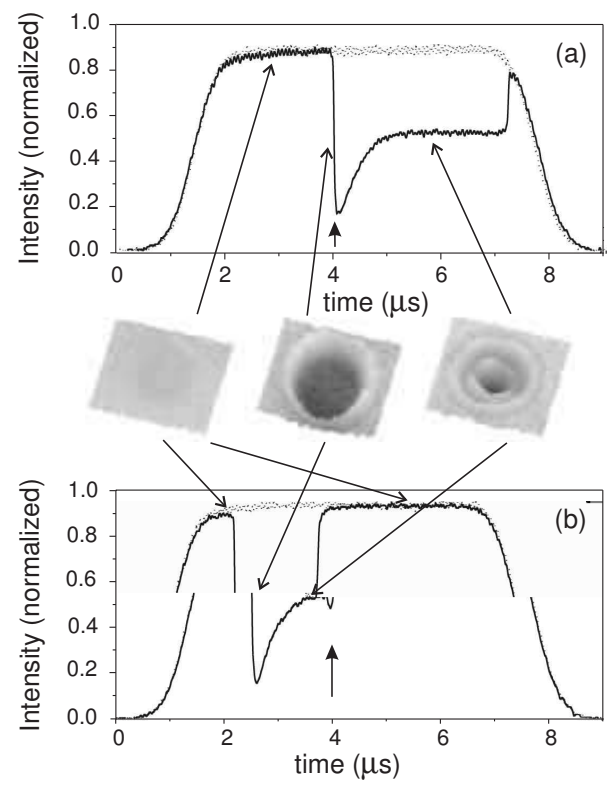

FIG. 23. Recording of incoherent switching-on (a) and switching-off (b) of a soliton. Snapshot pictures show unswitched state (left), circular switched domain (center) and a soliton (right). Dotted trace: incident intensity, solid trace: reflected intensity at center of soliton.

Besides the coherent switching (FIG. 15) bright solitons can also be switched by light incoherent with the background field. Under these conditions the switching occurs due to change of carrier density alone. FIG. 23 (a) shows incoherent switch-on of a bright soliton, where a 
perpendicularly polarized switching pulse of $\approx 10 \mathrm{~ns} \mathrm{du-}$ ration is applied at $t \approx 4.0 \mu \mathrm{s}$. As apparent, a soliton forms after this incoherent light pulse. The slow formation of the soliton is apparent in FIG. 23 (a) (using roughly the time from $t \approx 4.0 \mu \mathrm{s}$ to $t \approx 4.5 \mu \mathrm{s}$ ), indicating again the influence of material heating.

It should be emphasized that this heating is not instrumental for switching a soliton on. However, it allows switching a soliton off incoherently [32]. This is shown in FIG. 23 (b) where the driving light is initially raised to a level at which a soliton forms spontaneously. The slow soliton formation due to the heating is again apparent. The incoherent switching pulse is then applied which leads to disappearance of the soliton.

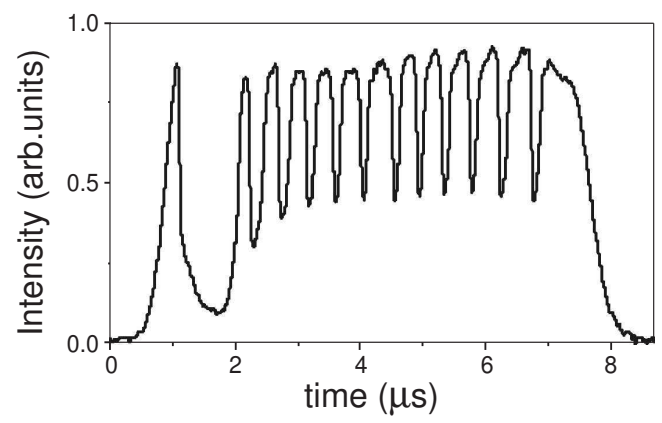

FIG. 24. Reflected light intensity measured at the center of a soliton showing the regenerative pulsing (repeated switching on and off of a soliton) resulting from combined thermal and electronic effects.

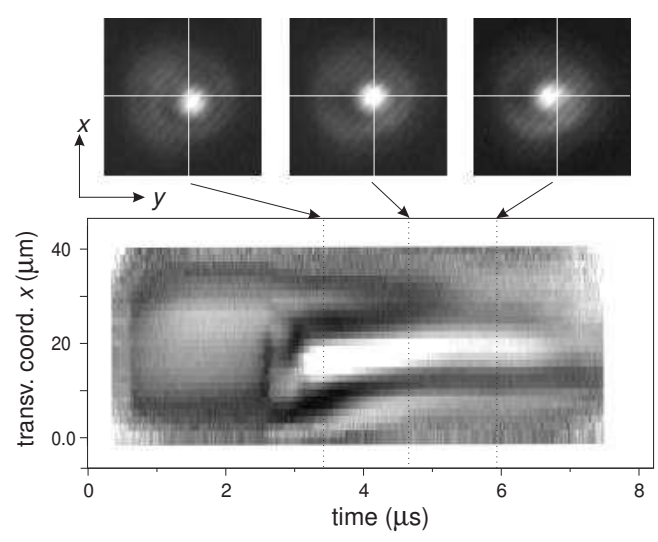

FIG. 25. Formation of a dark soliton (compare FIG. 22 and caption) and motion. The motion visible in the streak picture (bottom) is shown in 2D snapshots (top) for clarity.

The soliton can thus be switched on and also off by an incoherent pulse. The reason for the latter is thermal effect: Initially the material is "cold". A switching pulse leads then to the creation of a soliton. Dissipation in the material at the location of the soliton raises the temperature and the soliton is slowly formed. At the raised temperature the band edge (and with it the bistability characteristic and the existence range of solitons) is shifted so that a new pulse brings the system out of the range of existence of solitons. Consequently the soliton is switched off.

Thus switching on a soliton is possible incoherently with the "cold" material and switching off incoherently with the "heated" material. When the driving intensity is chosen to be slightly below the spontaneous switching threshold the nonlinear resonator is cold. An incoherent pulse increases the carrier density locally and can switch the soliton on, which causes local heating. Another incoherent pulse aimed into the heated area can then switch the soliton off and thereby return the resonator to its initial temperature, so that the soliton could be switched on/off again.

This thermal effect combined with electronic nonlinearity can cause spatial and temporal instabilities. A bright soliton switching on spontaneously in the cold material will then heat locally and can thereby destroy the condition for its existence, so that it switches off. After the material has cooled the soliton switches on again etc. Regenerative pulsing of the soliton results, an example of which is shown in the observation FIG. 24.

As opposed to the bright soliton that heats the material locally a dark soliton cools the material locally. This results in a shift of the band edge and with it the switching characteristic opposite to the bright soliton case. The consequence is that the dark soliton moves laterally to places of uncooled material. Here, however, it cools the material again so that a continuous motion results, analogously to what we have described as the "restless vortex" in [33]. Dark solitons in connection with material heating effects tend therefore to be non stationary. Such motion of a dark soliton was observed e.g. in [24] and is shown in FIG. 25.

\section{Illumination above bandgap (absorptive/focusing case)}

At excitation above bandgap bright solitons form [34] which have the same appearance as the bright solitons below/near band gap (Figs 14 (c) and 20 (a)).

FIG. 26 shows the dynamics of the bright soliton formation for excitation above bandgap. The difference between solitons existing above and below bandgap can be understood from the model. From (1) we obtain the reflected intensity as a function of incident intensity for wavelengths above the bandgap $(\operatorname{Re}(\alpha)>0)$, as well as below the bandgap $(\operatorname{Re}(\alpha)<0)$, for plane waves (FIG. 27). One sees that the bistability range is large below and small above the bandgap. Solving (1) numerically, the typical bright soliton (top of FIG. 27) is found coexisting with the homogeneous intensity solutions in the shaded regions of FIG. 27 (a), (b). 

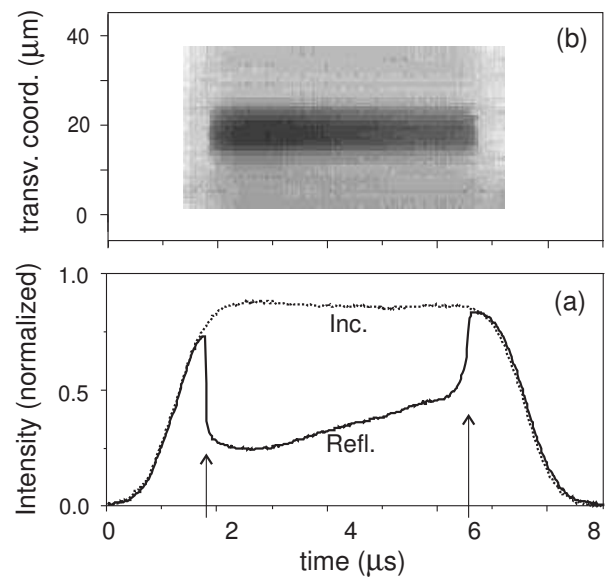

FIG. 26. Bright soliton formation above bandgap. Reflectivity on a diameter of the illuminated area as a function of time (b). Intensity of incident (dotted) and reflected (solid) light, at the center of the soliton as a function of time (a). Arrows mark the switch-on and -off. The soliton form fast (without mediation by a thermal effect as in FIG. 22).

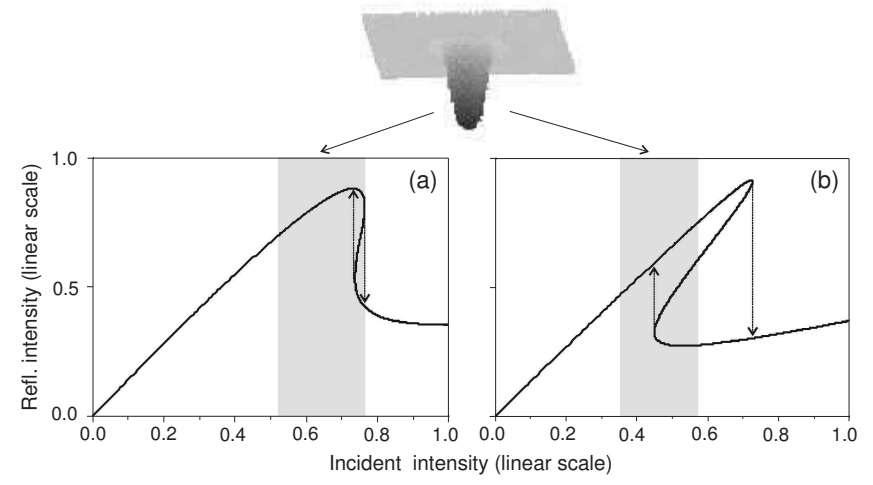

FIG. 27. Steady-state plane wave solution of Eq.(1) above bandgap (a) and below bandgap (b). The soliton solution shown exists for incident intensities corresponding to the shaded areas, in coexistence with the homogeneous solution. For a temperature increase the characteristics shift together with soliton existence ranges to higher incident intensities. Reflected and incident intensities normalized to the same value. Note the different widths of the bistability ranges above and below bandgap.

Above the band gap FIG. 26 shows that the soliton is switched on "immediately" without the slow thermal process described above. FIG. 27 (a) shows why. The plane wave characteristic of the resonator above band gap is either bistable in a very narrow range, or even monostable (due to the contribution of the self-focusing reactive nonlinearity [35]) but still with bistability between the soliton state (not plane wave) and the unswitched state. In this case the electronic switching leads directly into the basis of attraction for solitons and the switch-on of the soliton is purely electronic and fast. The widths of the bistability characteristics observed experimentally [34] correspond to the calculated ones FIG. 27. Conversely the wide bistability range below bandgap requires the (slow) thermal shift of the characteristic to reach the soliton existence range.

Nonetheless, also above bandgap there is strong dissipation after the switch-on. The associated temperature rise influences and can even destabilize a soliton. The destabilization effect can be seen in FIG. 26 (a). Over a time of a few $\mathrm{s}$ after the soliton switch-on the soliton weakens (reflectivity increases slowly) presumably by the rise of temperature and the associated shift of the band gap. At $6.5 \mu$ s the soliton switches off, although the illumination has not yet dropped.

Thus, while the dissipation does not hinder the fast switch-on of the soliton, it can finally destabilize the soliton. After the soliton is switched off, the material cools and the band gap shifts back so that the soliton can switch on again.

\section{Optical pumping}

The thermal effects discussed above result from the local heating caused by the high intracavity intensity within the bright soliton. They limit the switching speed of solitons and they will also limit the speed at which solitons could be moved around, thus limiting applications. The picture is that a soliton carries with it a temperature profile, so that the temperature becomes a dynamic and spatial variable influencing the soliton stability.

As opposed, a spatially uniform heating will not cause such problems, as it shifts parameters but does not constitute a variable in the system. The unwanted heating effects are directly proportional to the light intensity sustaining a soliton. For this reason and quite generally it is desirable to reduce the light intensities required for sustaining solitons.

Conceptually this can be expected if part of the power sustaining a resonator soliton could be provided incoherently with the driving field, e.g. by means of optical or electrical pumping. Pumping of the MQW structure generates carriers and allows conversion from absorption to gain. If pumping is strong enough the semiconductor microresonator can emit light as a laser [36].

FIG. 28 shows the variation of the plane-wave bistability domain with the pump as calculated from (1). The increase of the pump intensity leads to a shrinking of the bistability domain for plane waves (FIG. 28, pump intensities from $P_{1}$ to $P_{3}$ ) and the resonator solitons' existence domain while reducing the light intensity necessary to sustain the solitons. This reduction of the sustaining light intensity was observed experimentally (FIG. 29) [26]. When pumping below the transparency point of the material and with the driving laser wavelength near 
the semiconductor MQW structure band edge, bright and dark solitons form similarly to the unpumped case (FIG. 20). As the pump reduces the sustaining light intensity of the solitons, the heating effects are weak and the solitons switch on fast and unmediated by heating $[26]$.

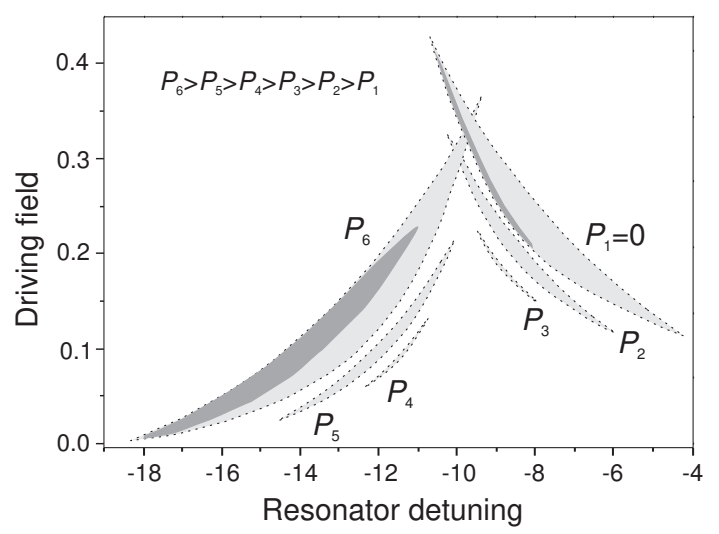

FIG. 28. Calculated plane-wave bistability domains as a function of the pump intensity. Dark shaded areas show bright soliton existence domains for two extreme cases: without pump $\left(P_{1}=0\right)$ and with pump near lasing threshold $\left(P_{6}\right)$.
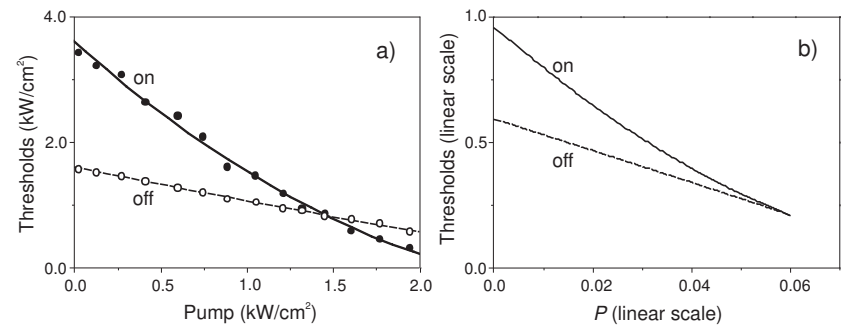

FIG. 29. Measured (a) and calculated (b) switch-on and switch-off intensities for the driving light as a function of the pump intensity. The unphysical crossing of the on and off curves is an artifact from material heating.

When the pump intensity approaches the transparency point of the semiconductor material, the resonator solitons' domain of existence disappears (FIG. 28, pump intensities between $P_{3}$ and $P_{4}$ ). It reappears above the transparency point (FIG. 28, pump intensities from $P_{4}$ to $P_{6}$ ) and expands with the pump intensity (FIG. 30).

Switched structures observed below the lasing threshold are shown in FIG. 31. FIG. 32 shows structures observed slightly above lasing threshold when a driving field is used simultaneously with the laser emission. The structures of FIG. 32 are reminiscent of the solitons in electrically pumped resonators [37]. It becomes clear that these structures (FIG. 32) must be soliton-collections or patterns, and not (linear) mode patterns, when looking at FIG. 32 (c). Here three bright spots are visibly separated by darker lines. In a "mode-pattern" the phase change from one bright spot to the next is $\pi$ (resulting in black lines due to destructive interference separating the bright spots). Therefore a "flower" mode pattern must have (and has) always an even number of "petals", whereas here we observe an odd number, inconsistent with phases in mode patterns. Thus we can conclude that there is no phase change between the bright spots and the latter are formed by self-localization, i.e. they are solitons or patterns in a resonator above transparency of the nonlinear material.

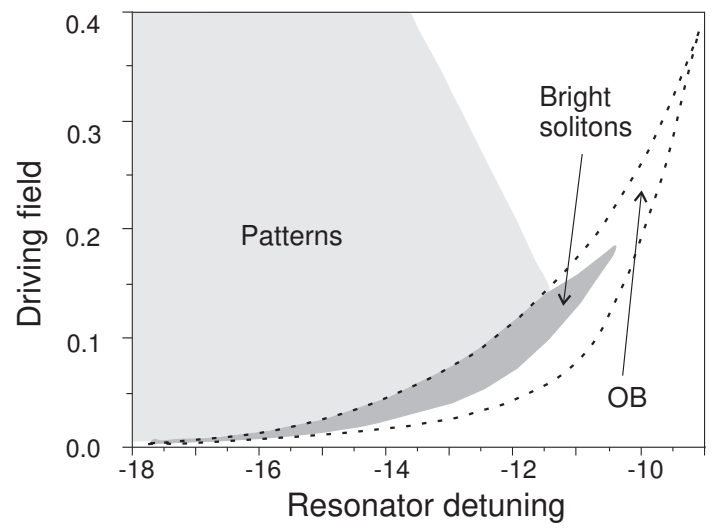

FIG. 30. Results of numerical simulations of below bandgap solitons using the model (1) for a microresonator pumped close to the lasing threshold. Shaded areas are domains of existence of bright resonator solitons and patterns. Area limited by dashed lines is optical bistability domain for plane waves.

We note that optically pumped resonators allow more homogeneous pumping conditions than electrical pumping [38]. This suggests that optical pumping lends itself more readily for localization and motion control of solitons than electrical pumping.

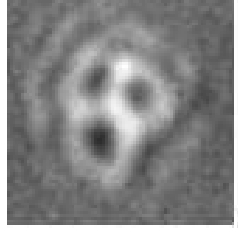

(a)

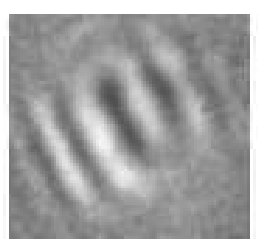

(b)

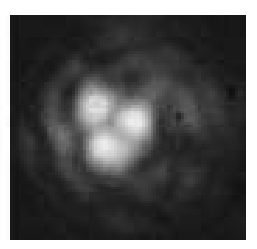

(c)
FIG. 31. Snapshots of typical optical structures at optical pump intensities slightly below lasing threshold (driving light intensity increases from (a) to (c)). With driving intensity dark spot patterns change to bright spot patterns.

There is a difference between resonator solitons below bandgap in pumped and unpumped material. The non- 
linear resonance mechanism of soliton formation [17] uses a defocusing nonlinearity below transparency and a focusing nonlinearity above transparency. Defocusing nonlinearity stabilizes dark solitons and focusing nonlinearity stabilizes bright solitons. It follows that dark solitons should prevail for unpumped material and bright solitons for pumped material. FIG. 30 shows typical examples of calculated resonator solitons for a pumped semiconductor microresonator. Bright solitons have a large existence range in the pumped case (FIG. 28, at $P_{6}$ ), dark solitons exist, though with smaller range of stability, in the unpumped case (FIG. 28, at $P_{1}$ ).

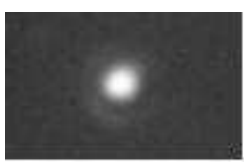

(a)

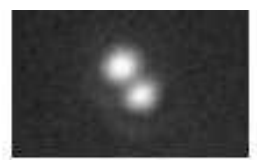

(b)

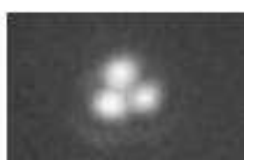

(c)
FIG. 32. Snapshots of typical optical structures at optical pump intensities slightly above lasing threshold (driving light intensity increases from (a) to (c)). Structures are always bright spot patterns.

Thus pumped semiconductor resonators are well suited for sustaining solitons below bandgap: (i) the background light intensity necessary to sustain and switch resonator solitons is substantially reduced by the pumping and therefore destabilizing thermal effects are minimized, (ii) the nonlinear resonance effect and the transverse nonlinear effect (self-focusing) cooperate to stabilize bright solitons, therefore the domain of existence of below bandgap (purely dispersive) bright solitons can be quite large.

\section{CONCLUSION}

We have shown in these experiments that optical solitons generally exist in nonlinear optical resonators. They can be vortices, phase solitons, or bright and dark solitons. For technical applications the experiments on semiconductor microresonators have shown the existence of bright and dark solitons. One can experimentally distinguish switched areas, patterns and solitons from each other and from mode-fields (i.e. fields whose structure is standing transverse waves resulting from boundaries). Thermal effects can lead to spatial and temporal instabilities. They can be controlled in various ways e.g. by working above bandgap or by pumping. Pumping allows furthermore to control the magnitude and the sign of the material nonlinearities, and thus to maximize stability ranges for bright (dark) solitons. Coherent periodic patterns can disintegrate with increasing driving field into independent spatial solitons.
Optical resonator solitons show properties reminiscent of simple biological structures and one may speculate that aggregations of resonator solitons could allow information processing reminiscent of brain functions.

Acknowledgment

This work was supported by Deutsche Forschungsgemeinschaft under grant We743/12-1.

[1] W.J. Firth, A.J. Scroggie: Europhys. Lett. 26, 521 (1994)

[2] U. Frisch: Turbulence. The Legacy of A.N.Kolmogorov, Cambridge University Press (1995)

[3] K. Staliunas: Int. Journ. of Bifurcation and Chaos 11, 2845 (2001)

[4] K. Staliunas: "Anticorrelation and Subdiffusion in Financial Systems", xxx.lanl.gov, cond.mat/0203591 (2002); subm. Phys. Rev. E (2002)

[5] For a review see e.g. P. Dutta, P. M. Horn: Rev. Mod. Phys. 53, 497 (1981)

[6] P. Bak: Phys. Rev. Lett. 59, 381 (1987)

[7] See as examples: A. Jaques, P. Glorieux: Opt. Comm. 40, 455 (1982) and S. Rushin, S.M. Bauer: Appl. Phys. 24, 45 (1981)

[8] V.B. Taranenko, C.O. Weiss, B. Schäpers: Phys. Rev. A 65, 013812 (2002)

[9] G. Slekys, K. Staliunas, C.O. Weiss: Opt. Comm. 149, 113 (1998)

[10] C.O. Weiss, M. Vaupel, K. Staliunas, G. Slekys, V.B. Taranenko: Appl. Phys. B 68, 151 (1999)

[11] See the supplementary electronic material to [10]: http://link.springer.de/jounals/apb

[12] C.P. Smith, Y. Dihardja, C.O. Weiss, L.A. Lugiato, F. Prati, P. Vanotti: Opt. Comm. 102, 105 (1999)

[13] D. Oesterhelt, W. Stoekenius: Nature 233, 149 (1971)

[14] V.Yu Bazhenov, V.B. Taranenko, M.V. Vasnetsov: Proc. SPIE, 1840, 183 (1992)

[15] V.B. Taranenko, K. Staliunas, C.O. Weiss: Phys. Rev. A 56, 1582 (1997)

[16] K. Staliunas, V.B. Taranenko, G. Slekys, R. Viselga, C.O. Weiss: Phys. Rev. A 57, 599 (1998)

[17] K. Staliunas, V.J. Sanchez-Morcillo: Opt. Comm. 139, 306 (1997)

[18] V.B. Taranenko, K. Staliunas, C.O. Weiss: Phys. Rev. Lett. 81, 2226 (1998)

[19] H.M. Gibbs: Optical Bistability - Controlling Light with Light, Academic Press, (1985)

[20] L. Spinelli, G. Tissoni, M. Brambilla, F. Prati, L.A. Lugiato: Phys. Rev. A 58, 2542 (1998)

[21] D. Michaelis, U. Peschel, F. Lederer: Phys. Rev. A 56, R3366 (1997)

[22] L.A. Lugiato, R. Lefever: Phys. Rev. Lett. 58, 2209 (1987)

[23] N.N. Rosanov:, Prog. Opt. 35, 1 (1996)

[24] V.B. Taranenko, I. Ganne, R. Kuszelewicz, C. O. Weiss: Phys. Rev. A 61, 063818 (2000) 
[25] V.B. Taranenko, F.-J. Ahlers, K. Pierz: Appl. Phys. B 75, 75 (2002)

[26] V.B. Taranenko, C.O. Weiss, W. Stolz: Opt. Lett. 26, $1574(2001)$

[27] V.B. Taranenko, I. Ganne, R. Kuszelewicz, C. O. Weiss: Phys. Rev.A 61, 063818 (2000)

[28] P.K. Jacobsen, J.V. Moloney, A.C. Newell, R. Indik: Phys. Rev. A 45, 8129 (1992)

[29] W.J. Firth, A.J. Scroggie: Europhys. Lett. 26, 521 (1994)

[30] G.I. Stegeman, M. Segev, Science 286, 1518 (1999)

[31] T. Rossler, R.A. Indik, G.K. Harkness, J.V. Moloney, C.Z. Ning: Phys. Rev. A 58, 3279 (1998)

[32] V.B. Taranenko, C.O. Weiss: Appl. Phys. B 72, 893 (2001)

[33] C.O. Weiss, H.R. Telle, K. Staliunas, M. Brambilla: Phys. Rev. A 47, R1616 (1993)

[34] V.B. Taranenko, C.O. Weiss, W. Stolz: J. Opt. Soc. Am. B 19, 8129 (2002)

[35] S.H. Park, J.F. Morhange, A.D. Jeffery, R.A. Morgan, A. Chavez-Pirson, H.M. Gibbs, S.W. Koch, N. Peyghambarian, M. Derstine, A.C. Gossard, J.H. English, W. Weidmann: Appl. Phys. Lett. 52, 1201 (1988)

[36] V.B. Taranenko, C.O. Weiss: "Spatial solitons in an optically pumped semiconductor microresonator", xxx.lanl.gov, nlin.PS/0204048 (2002)

[37] Report as given in www.pianos-int.org

[38] W.J. Alford, T.D. Raymond, A.A. Allerman: J. Opt. Soc. Am. B 19, 663 (2002) 